\title{
Nonlinear Control Design for a Plasma Process
}

\author{
Petar Iordanov, John Ringwood and Seán Doherty
}

\begin{abstract}
This paper presents a nonlinear control design for a first-principles based model of an argon plasma process. In this study, a Hammerstein-type structure was employed as a basis for a feedback control design. Artificial neural networks were used to accurately model the static nonlinearity. In the developed Hammerstein model, variations in the process dynamics were accounted for by considering parametric uncertainty. A control design strategy based on $\mu$-synthesis was applied to deliver good tracking performance and disturbance rejection.
\end{abstract}

\section{INTRODUCTION}

In recent years, the reactive ion etching (RIE) process has received tremendous attention and nowadays it has no equal in value alternative in the semiconductor industry. RIE is a low-pressure plasma system, which involves chemical and physical reactions to achieve an etching process with suitable properties such as selectivity, uniformity and anisotropy. The physical and chemical mechanisms in RIE are known to be very complex, and are currently not entirely understood.

The modelling of plasma processes requires a thorough understanding of the plasma dynamics, which is naturally hard to analyse. This process is known to be sensitive to various parameters, such as chamber geometry, accuracy of measurement sensors, chemical disturbances, etc., [1]. In other words, a data-based model obtained for a particular plasma chamber would not necessarily be good enough for another chamber of the same brand and specifications, exploited under the same operating conditions. A more sophisticated approach to model plasma processes is to exploit the information that is available about the physical and chemical interactions that occur in the process. Models based on this methodology are referred to as first-principles models. As opposed to data-based models, first-principles models take advantage of parameters that describe chamber geometry, delivery of radio-frequency (RF) power to the chamber, actuators, etc. While a lot of work has been done on first-principles based models for RIE, various literature sources have pointed out that these models are not suitable for analysis and control system design, [2]. Although such models are typically quite complex and may exhibit severe nonlinearities, once fully developed, they can be applied for control design of any plasma system designated for RIE.

Petar Iordanov is with Department of Electronic Engineering, National University of Ireland at Maynooth, Maynooth, Republic of Ireland. petar.iordanov@eeng.nuim.ie

John Ringwood is with Department of Electronic Engineering, National University of Ireland at Maynooth, Maynooth, Republic of Ireland. john.ringwood@eeng. nuim. ie

Seán Doherty is with Department of Electronic Engineering, National University of Ireland at Maynooth, Maynooth, Republic of Ireland. sean.doherty@eeng.nuim.ie

This work was supported by Science Foundation Ireland, SFI.
Model complexity (model order) can be reduced through eliminating static and dynamic components that have none or very little effect on the plasma model behaviour. However, parametric variations and neglected dynamics can be accommodated in the model as structured and/or unstructured model perturbations. Although the majority of control design techniques are based on linear models, such models are only adequate for a relatively small operating space. Since real process models are essentially nonlinear, it is more natural to use nonlinear models for control design. Despite the fact that such models are typically difficult to analyse, they provide more accurate system representation of the process and allow exploiting larger operating space. In recent years, nonlinear model structures based on separation of static and dynamic responses have become quite popular. However, such separation is not always possible. Models of this type are normally referred as to Wiener and Hammerstein structures. In Hammerstein structures, which are preferred by researchers, the memoryless static nonlinearity is followed by a linear dynamic block, as shown in Fig.1. Such decomposition facilitates the feedback design procedures and provides valuable information regarding the system characteristics. Plasma processes used for RIE can be modelled as Hammerstein systems, under certain operating conditions. It should be noted that neural network based models and Hammerstein systems are widely used in modelling and control of RIE processes, [2]-[6]. However, these models are identified using data-based approaches, which do not take advantage of the information that first-principles models provide. Using a first-principles model, parametric uncertainty in the linear dynamical model can be easily estimated. An appropriate control design tool to deal with this type of uncertainty is $\mu$-synthesis, [7], [8].

The use of artificial neural networks and Hammerstein structure to describe a first-principle based model, is considered in this paper. The Hammerstein model developed in this study is exploited in conjunction with a robust control methodology to achieve satisfactory closed-loop performance.

The paper is organised as follows: Section II gives a brief description of the first-principles based model of the plasma process, including some notable features of the model. Development of a Hammerstein model that supports structured model perturbations, is presented in section III. A $\mu$-synthesis controller design that addresses uncertainty in the process dynamics and possible modelling inaccuracy in the static nonlinearity, is detailed in section IV. Finally, conclusions and future research directions are outlined in section $\mathrm{V}$. 


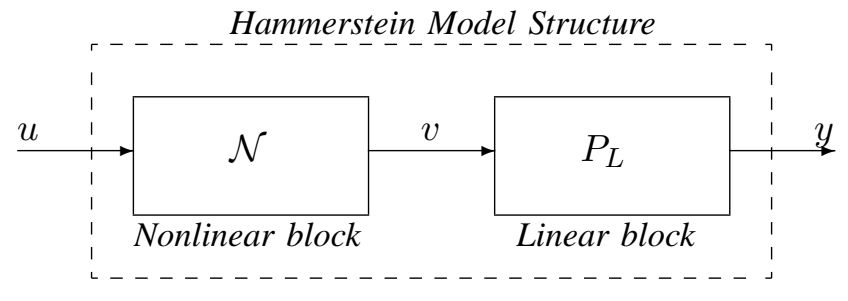

Fig. 1. Hammerstein Model Block Diagram

\section{PLASMA PROCESS MODEL}

\section{A. Model Description}

Description of a global model of an argon plasma process is now presented. Here, global means that spatial gradients within the chamber are not considered, so that the concentration of each chemical species inside the chamber is described by a single state variable. The process itself is a one-species plasma confined in a cylindrical chamber. The model is based on known physical interactions that are present in the plasma. Four nonlinear differential equations describe the dynamics of the process, [9]-[11]:

$$
\begin{aligned}
& \frac{d n}{d t}=k_{i} N n-\frac{A}{V} \Gamma_{n} \\
& \frac{d N}{d t}=\frac{F}{M V}-\frac{A_{E} \Gamma_{N}}{V}-k_{i} N n+\frac{\left(A-A_{E}\right) \Gamma_{n}}{V} \\
& \frac{d}{d t}\left(\frac{3}{2} n k_{B} T_{e}\right)=\frac{P}{V}-\epsilon_{i} k_{i} N n-\epsilon_{*} k_{*} N n- \\
& \quad \frac{3}{2} \delta\left(k_{B} T_{e}-k_{B} T_{g}\right) k_{e} N n- \\
& \quad \frac{A}{V}\left[\frac{5}{2}+\frac{1}{2} \ln \left(\frac{M}{2 \pi m}\right)\right] k_{B} T_{e} \Gamma_{n} \\
& \frac{d}{d t}\left(\frac{3}{2} N k_{B} T_{g}\right)=\frac{3}{2} \frac{F}{M V} k_{B} T_{i n}-\frac{3}{2} k_{B} T_{g} \frac{A_{E}}{V} \Gamma_{N}+ \\
& \quad \frac{3}{2} \delta\left(k_{B} T_{e}-k_{B} T_{g}\right) k_{e} N n+ \\
& \quad \frac{3}{2} \gamma \frac{\left(A-A_{E}\right)}{V}\left(k_{B} T_{w}-k_{B} T_{g}\right) \Gamma_{N}
\end{aligned}
$$

Equations (1)-(4) are, respectively, balance equations for charged particles, neutral density, electron temperature and gas temperature. These equations describe the dynamics of the following state variables:

$$
\begin{aligned}
n & - \text { electron/plasma density } \\
N & - \text { neutral density } \\
T_{e} & - \text { electron temperature } \\
T_{g} & - \text { temperature of other gas species }
\end{aligned}
$$

Control of the process is established through the following manipulated variables:

$$
\begin{aligned}
F & - \text { gas flow rate } \\
P & - \text { RF power } \\
A_{E} & - \text { exhaust port area }
\end{aligned}
$$

Measured variables are the ion flux (charged particle flux), $\Gamma_{n}$, and the neutral flux, $\Gamma_{N}$. Both, ion flux and neutral flux, are nonlinear functions of the state variables, and typical expression for them are:

$$
\begin{aligned}
\Gamma_{n} & =n h \sqrt{\frac{k_{B} T_{e}}{M}} \\
\Gamma_{N} & =\frac{N}{4} \sqrt{\frac{8 k_{B} T_{g}}{\pi M}}
\end{aligned}
$$

The controlled variable of interest is the ion flux, $\Gamma_{n}$, which is explicitly related to the etch rate in RIE reactors. A list of the parameters involved in equations (1)-(4) is given in Table I.

TABLE I

PLASMA MODEL PARAMETERS

\begin{tabular}{|l|l|}
\hline Parameter & Description \\
\hline$A$ & Internal surface area of the chamber \\
$h$ & Volume of the chamber \\
& Parameter that accounts for \\
$M$ & reduction on $n$ near the walls \\
$m$ & Neutral mass \\
$\delta$ & Electron mass \\
$\epsilon_{i}$ & Mass ratio, $2 m / M$ \\
$\epsilon_{*}$ & Ionisation energy \\
$k_{B}$ & Excitation energy \\
$k_{e}$ & Boltzman constant \\
$k_{i}$ & Energy rate \\
$k_{*}$ & Ionisation rate, $k_{i}=k_{i}^{(0)} e^{-\left(\frac{\epsilon_{i}}{k_{B} T_{e}}\right)}$ \\
$\gamma$ & Excitation rate, $k_{*}=k_{*}^{(0)} e^{-\left(\frac{\epsilon_{*}}{k_{B} T_{e}}\right)}$ \\
$T_{w}$ & Accommodation coefficient, $\gamma \in[0,1]$ \\
$T_{i n}$ & Temperature of chamber wall \\
\hline
\end{tabular}

In this study, several assumptions related to the description of the current plasma model are taken into account, [9]. Firstly, the approximation that all species other than electrons have the same temperature is justified if there are sufficiently frequent collisions that share energy between particles of different species. Secondly, it is assumed that there are no losses in delivering RF power to the chamber, i.e. power deposition model is not considered. In addition, actuators are not presently included in the problem formulation. The complexity of the power deposition model and the actuators, and their influence on the process behaviour is a subject of further development.

\section{B. Model Characteristics}

A notable feature of the plasma model under consideration is the presence of Arrhenius type of nonlinearities (terms like $e^{-E / R T}$ ). Such nonlinearities are "severe" and complicate the analytical computation of steady-state solutions. For the model described by (1)-(4), state equilibria was determined through numerical simulations. It should be noted that at each operating point, defined by $\left\{F_{i}, P_{i}, A_{E i}\right\}$, only one plausible steady-state solution, $\left\{n^{\circ}, N^{\circ}, T_{e}^{\circ}, T_{g}^{\circ}\right\}$, can be located. Another feature of the system of differential equations is their stiff nature. For the considered model, at certain operating points the ratio of the largest and smallest time constants can be as large as $10^{5}$. 
The above discussed features suggest that the plasma process model given by (1)-(4), is inappropriate for model-based control design. Analysis has shown that in the following operating range

$$
\begin{aligned}
& F \in\left[10^{-5}, 10^{-4}\right] \mathrm{kg} / \mathrm{s} \\
& P \in[400,1200] \mathrm{W} \\
& A_{E}=10^{-2} \mathrm{~m}^{2}
\end{aligned}
$$

variations in the dynamics of both $\Gamma_{n}$ and $\Gamma_{N}$, are not significant. Consequently, the plasma model in this operating range can effectively be approximated by a Hammerstein structure. In the present study, $A_{E}$ is constant, and consequently is not considered as a manipulated variable. The development of the Hammerstein model is discussed in the next section.

\section{MODELLING FOR CONTROL DESIGN}

A model-based control strategy is considered in this work, [6]. The closed-loop system interconnection is presented in Fig.2. The main objective of this structure is to linearise the open-loop process. In the block diagram, the plasma model has a Hammerstein structure, where $\mathcal{N}$ is the memoryless static nonlinearity and $P_{L}$ is the linear dynamic block. The controller device, $K=\mathcal{N}^{-1} K_{L}$, consists of a linear controller $K_{L}(s)$ and the inverse model nonlinearity, $\mathcal{N}^{-1}$. If $\mathcal{N}^{-1}$ is an exact inverse of $\mathcal{N}$, then there will be an identity mapping from $K_{L}$ to $P_{L}$.

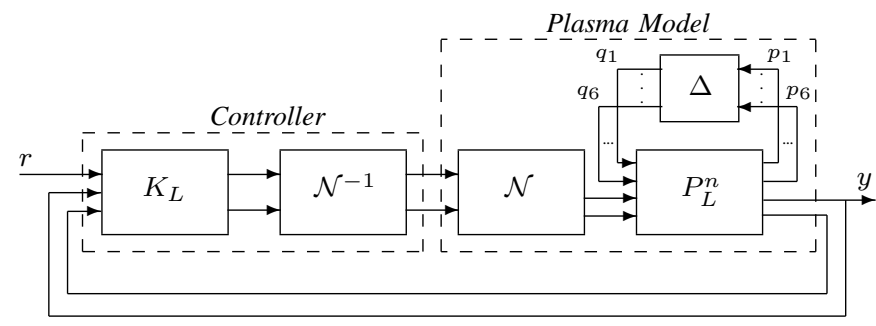

Fig. 2. Ion flux control structure

The use of feedforward backpropagation neural networks was employed to model both $\mathcal{N}$ and $\mathcal{N}^{-1}$. The nonlinearity $\mathcal{N}$ was modelled by using 10 neurons in the only hidden layer, while $\mathcal{N}^{-1}$ was approximated by 20 neurons. Hyperbolic tangent sigmoid transfer functions (tansig) were used in the hidden layer. The accuracy of the resulting approximations were tested using the following indicators:

$$
\begin{aligned}
& \Delta F(F, P)=\frac{|F-\tilde{F}|}{F} \times 100 \% \\
& \Delta P(F, P)=\frac{|P-\tilde{P}|}{P} \times 100 \%
\end{aligned}
$$

where

$$
\left[\begin{array}{l}
\tilde{F} \\
\tilde{P}
\end{array}\right]=\mathcal{N N}^{-1}\left[\begin{array}{l}
F \\
P
\end{array}\right]
$$

Here, $\Delta F$ and $\Delta P$ were computed at a grid of different operating points spanning the operating space. Ideally, $\mathcal{N} \mathcal{N}^{-1}=I$, which would ensure that $\Delta F=0$ and $\Delta P=0$. A grid of 400 operating points, $\left\{F_{i}, P_{i}\right\}$, was generated for this analysis. At all tested operating points, $\Delta P<0.4 \%$ and $\Delta F<3 \%$. The imperfection of the nonlinear model given by (1)-(4) and the inaccuracy in the approximation of the static nonlinearity was addressed by introducing parametric uncertainty in the linear block. The linear part of the Hammerstein model is a $2 \times 2$ transfer matrix $P_{L}(s)$, which can be expressed as an upper linear fractional transformation (denoted by $\mathcal{F}_{u}$ ) of $P_{L}^{n}(s)$ with respect to the perturbation $\Delta$, i.e.,

$$
\begin{aligned}
P_{L}(s)= & \mathcal{F}_{u}\left(P_{L}^{n}(s), \Delta\right) \\
\Delta= & {\left[\begin{array}{cccc}
\delta_{1} & 0 & \ldots & 0 \\
0 & \delta_{2} & \ldots & \vdots \\
\vdots & \vdots & \ddots & \vdots \\
0 & 0 & \ldots & \delta_{6}
\end{array}\right],\left|\delta_{i}\right| \leq 1 }
\end{aligned}
$$

Here, $P_{L}^{n}(s)$ denotes the nominal linear model, while $\delta_{i}$ 's represent the uncertainty in the time constants $\tau_{1}$ and $\tau_{2}$, and gains $k_{11}, k_{12}, k_{21}$ and $k_{22}$. The linear block $P_{L}(s)$ has a general form given by

$$
P_{L}(s)=\left[\begin{array}{cc}
\frac{k_{11}}{\tau_{1} s+1} & \frac{k_{21}}{\tau_{2} s+1} \\
\frac{k_{12}}{\tau_{1} s+1} & \frac{k_{22}}{\tau_{2} s+1}
\end{array}\right]
$$

where

$$
\begin{aligned}
\tau_{1} & =\bar{\tau}_{1}\left(1+w_{1} \delta_{1}\right) \\
\tau_{2} & =\bar{\tau}_{2}\left(1+w_{2} \delta_{2}\right) \\
k_{11} & =\bar{k}_{11}\left(1+w_{3} \delta_{3}\right) \\
k_{21} & =\bar{k}_{21}\left(1+w_{4} \delta_{4}\right) \\
k_{12} & =\bar{k}_{12}\left(1+w_{5} \delta_{5}\right) \\
k_{22} & =\bar{k}_{22}\left(1+w_{6} \delta_{6}\right)
\end{aligned}
$$

In this notation, $\bar{\tau}_{1}, \bar{\tau}_{2}, \bar{k}_{11}, \bar{k}_{12}, \bar{k}_{21}$ and $\bar{k}_{22}$ are the nominal values of the parameters, while $w_{i}$ 's represent the level of parametric uncertainty. A block diagram of the nominal model, $P_{L}^{n}(s)$, is shown in Fig.3. Here, the constant matrices $M_{1}$ and $M_{2}$ are:

$$
M_{1}=\left[\begin{array}{cc}
\bar{\tau}_{1}^{-1} & -w_{1} \\
\bar{\tau}_{1}^{-1} & -w_{1}
\end{array}\right], \quad M_{2}=\left[\begin{array}{cc}
\bar{\tau}_{2}^{-1} & -w_{2} \\
\bar{\tau}_{2}^{-1} & -w_{2}
\end{array}\right]
$$

In the figure, $p_{i}$ and $q_{i}$ denote the inputs and outputs of the structured perturbation $\Delta$. The nominal model was generated at the operating point $\left\{F=5.5 \times 10^{-5}, P=800\right\}$, which corresponds to $\Gamma_{n}=6.05 \times 10^{20}$ at DC. The parameters $w_{1}$ and $w_{2}$, representing bounds on the time constants, were obtained from time-domain analysis, which includes generation of step responses at various operating points. In this analysis, ranges of variation in step magnitudes for flow rate and power were $\left[10^{-6}, 10^{-5}\right] \mathrm{kg} / \mathrm{s}$ and $[10,100] \mathrm{W}$, respectively. The weights $w_{3}, \ldots, w_{6}$ cater for an uncertainty level of up to $30 \%$ in the effective static nonlinearity reflected by (1)-(4). The identified parameters of the linear model are presented in Table II. 


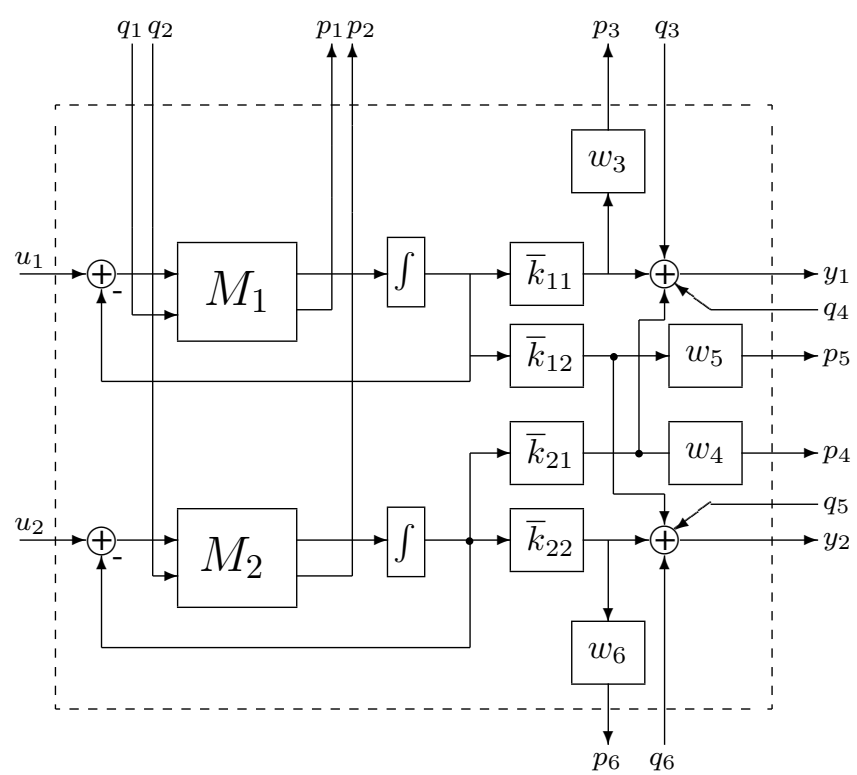

Fig. 3. Block diagram of the nominal linear model

TABLE II

PARAMETERS OF THE LINEAR MODEL

\begin{tabular}{|l|l|}
\hline Parameter & Value \\
\hline $\bar{\tau}_{1}$ & $2.87 \times 10^{-3} s$. \\
$\bar{\tau}_{2}$ & $1.1 \times 10^{-3} s$. \\
$\bar{k}_{11}$ & 0.863 \\
$\bar{k}_{21}$ & 0.124 \\
$\bar{k}_{12}$ & 0.951 \\
$\bar{k}_{22}$ & 0.171 \\
$w_{1}$ & $0.285(=28.5 \%)$ \\
$w_{2}$ & $0.23(=23 \%)$ \\
$w_{3}$ & $0.3(=30 \%)$ \\
$w_{4}$ & $0.3(=30 \%)$ \\
$w_{5}$ & $0.3(=30 \%)$ \\
$w_{6}$ & $0.3(=30 \%)$ \\
\hline
\end{tabular}

\section{CONTROLLER DESIGN}

The next step after development of a Hammerstein model, is to design a linear controller $K_{L}$ that stabilises the linear portion of the plant and provides satisfactory closedloop performance in the face of the specified parametric uncertainty. The conventional $\mu$-synthesis controller design strategy, [7], [8], also known as the $D-K$ iteration, was applied in this work. This robust control strategy naturally addresses design problems involving dynamic and parametric model perturbations. The $\mathrm{D}-\mathrm{K}$ iteration approach is based on the structured singular value metric, [12], referred to as $\mu$.

For this design, uncertainty in the time-constants, $\tau_{1}$ and $\tau_{2}$, and gains $k_{11}, k_{12}, k_{21}$ and $k_{22}$, is considered. To ensure robustness to parametric uncertainty, the objective is to find a controller $K_{L}$ that maximises

$$
\min _{i}\left|\delta_{i}\right|, \quad \delta_{i} \in \mathcal{R}, i=1, \ldots, 6
$$

while retaining closed-loop stability. Performance weights are introduced to define the desired closed-loop performance criteria. The robust control design structure is shown in Fig.4.

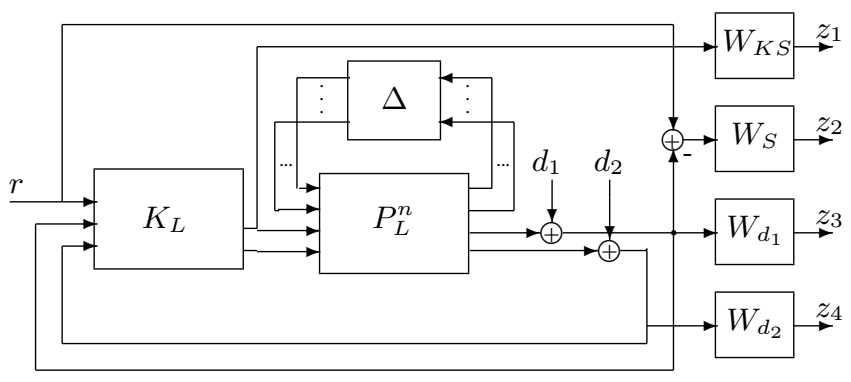

Fig. 4. Robust control design structure

In the block diagram, $r$ is the reference for $\Gamma_{n}$, while $d_{1}$ and $d_{2}$ are disturbances in $\Gamma_{n}$ and $\Gamma_{N}$, respectively. The spectrum of the disturbances are modelled by the weighting filters $W_{d 1}(s)$ and $W_{d 2}(s)$. The desired closedloop performance is to achieve a tracking error at DC of less than $3 \%$, no overshoot, and a settling-time of less than $0.2 s$. This requirement is specified by the filter $W_{S}(s)$. The weight $W_{K S}$ was introduced to minimise the mass flow rate. For the purpose of presentation, let $T_{x y}(s)$ denote the transfer function from the input $x$ to the output $y$. Then, the performance transfer matrix can be defined as

$$
H(s)=\left[\begin{array}{c}
T_{r z_{1}}(s) \\
T_{r z_{2}}(s) \\
T_{d_{1} z_{3}}(s) \\
T_{d_{2} z_{4}}(s)
\end{array}\right]
$$

The control design objective is to produce a stabilising controller $K_{L}(s)$ such that, for all admissible perturbations $\Delta$, with $\|\Delta\| \leq 1$, the perturbed closed-loop system remains stable, and the perturbed weighted performance transfer function, $H(s)$, has an $\mathcal{H}_{\infty}$ norm of less than unity for all such perturbations. These mathematical objectives exactly fit in the $\mu$ framework. Note that robust stability and performance specifications are satisfied if $\mu<1$. For the following selection of weighting filters and a single $\mathrm{D}-\mathrm{K}$ iteration, a robust performance of $\mu=0.96$ was achieved.

$$
\begin{array}{r}
W_{K S}=30 \quad, \quad W_{S}(s)=\frac{2000}{6 s+1} \\
W_{d_{1}}(s)=\frac{15}{10 s+1} \quad, \quad W_{d_{2}}(s)=\frac{3}{300 s+1}
\end{array}
$$

A constant scaling matrix was used in the synthesis, which resulted in a low-order controller, $K_{L}$, described by 5 states.

Closed-loop transient responses are shown in Fig. 5, where the step sequence was arbitrarily chosen. For this analysis, the original model described by (1)-(4) was used as a plant model. A more detailed look at the transient behaviour can be seen in Fig.6. The response indicates a negligible steadystate error and a settling time of less than $0.2 s$. The flow rate and power signals that correspond to the output $\Gamma_{n}$ in Fig.5, are shown in Fig.7 and Fig.8. It can be seen from the figures that control force is mainly provided by the power. As a result of the minimisation of $T_{r z_{1}}$, which is virtually equivalent to minimisation of the gas consumption, the gas flow rate was set to its minimum operating point, 
$F=10^{-5} \mathrm{~kg} / \mathrm{s}$. Figures indicate good performance well away from the nominal model (represented by a dashed line in Fig. 5, 7 and 8), thus highlighting the robustness of the design. Rejection to input and output disturbances is illustrated in Fig.9. In this figure, step changes in flow rate, power and ion flux were applied at $t=3 s, t=4 \mathrm{~s}$ and $t=5 s$, respectively. The three responses are indicated in the figure by 1,2 and 3 .

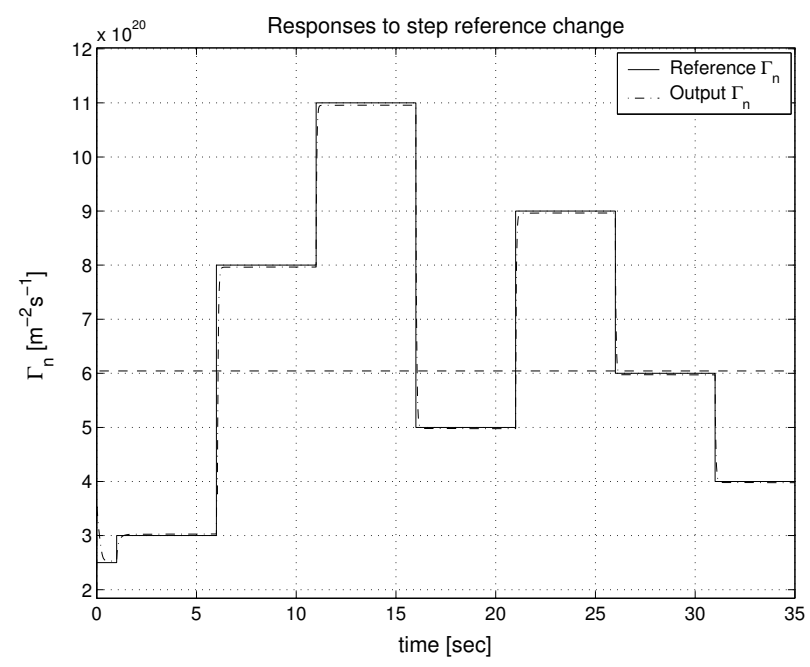

Fig. 5. Closed-loop response to ion flux reference trajectory

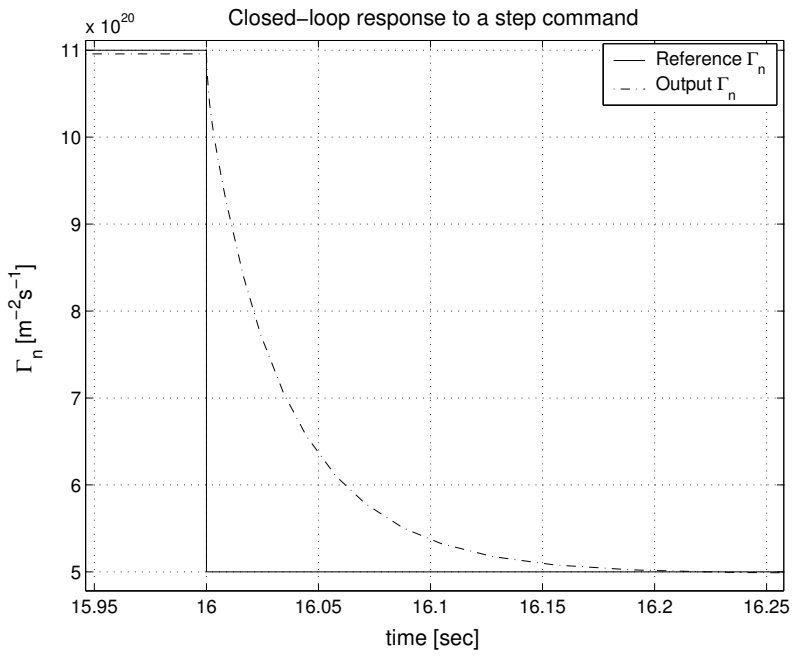

Fig. 6. A detailed view of the closed-loop step response

\section{CONCLUSIONS AND FUTURE WORK}

In this paper, a control of a first-principle based model of an argon plasma process has been considered. The differential equations that describe the model contain severe nonlinearities and exhibit stiff nature, which make the process model inappropriate for many feedback design procedures. To facilitate the design, a Hammerstein model that accounts for variations in the process dynamics has been derived. A model-based control design strategy was exploited in this

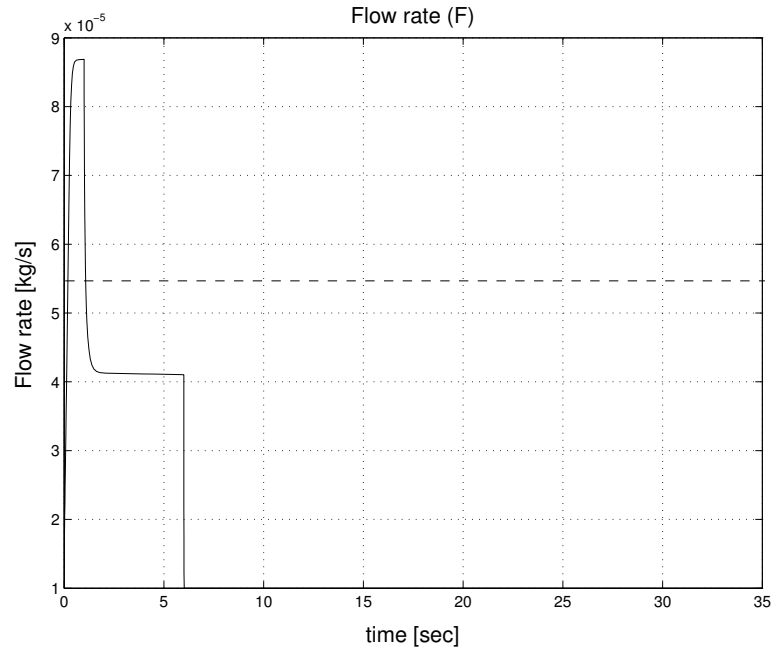

Fig. 7. Flow rate control signal

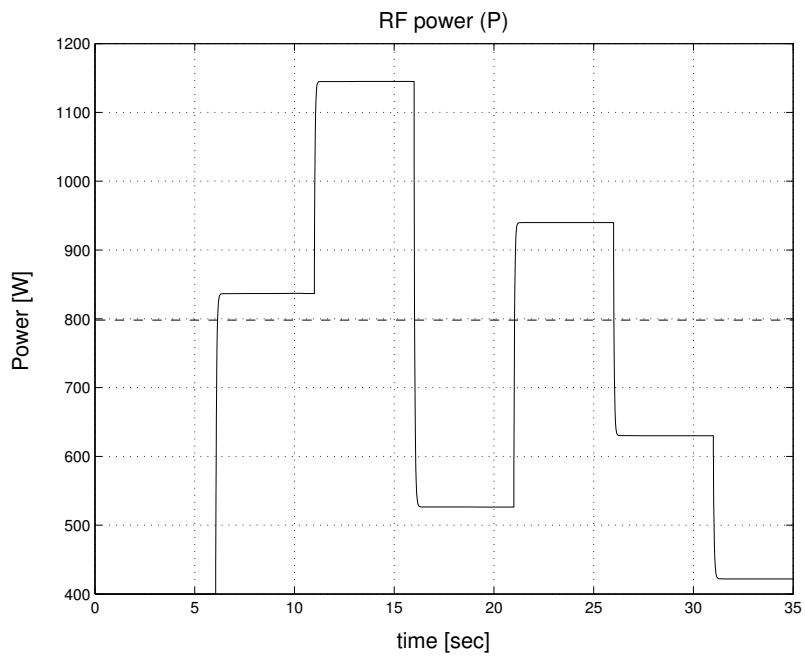

Fig. 8. Power control signal

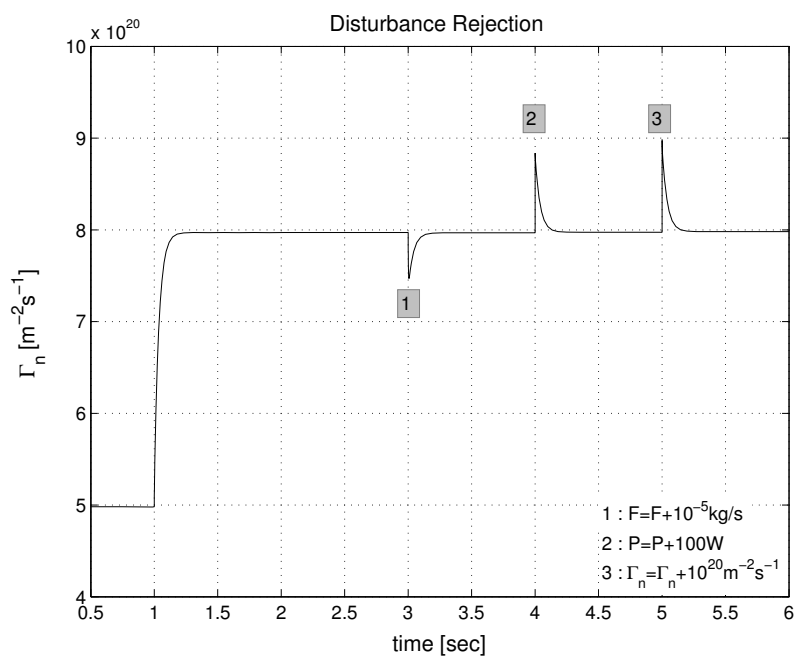

Fig. 9. Responses to input and output disturbances 
study, where parametric uncertainty was readily addressed by the conventional $\mu$-synthesis approach. The synthesised nonlinear controller is of low complexity, and provides excellent performance in both command tracking and disturbance rejection.

The present work is an attempt to investigate the use of first-principle based plasma models for controller design. Although this research is in its early stage, such results can be of tremendous help as progress advances. As the complexity of the studied models will increase, future work will focus on the development of control strategies that will ensure higher production efficiency in the semiconductor manufacturing, in particular, plasma etching.

\section{ACKNOWLEDGMENTS}

This work has been undertaken within the PlasMAC project, a joint collaboration between National University of Ireland at Maynooth and Dublin City University, and supported by Science Foundation Ireland (SFI).

\section{REFERENCES}

[1] T.A. Badgwell, T. Breeduk, S.G. Bushman, S.W. Butler, S. Chatterjee, T.F. Edgar, A.J. Toprac and I. Trachtenberg, Modelling and control of microelectronics material processing. Computers Chemical Engineering, Vol. 19, 1995, pp. 1-41.
[2] T.L. Vincent, P.P. Khargonekar, B.A. Rashap, F. Terry and M. Alta, "Nonlinear system identification and control of a reactive ion etcher", Proceedings of the American Control Conference, Baltimore, Maryland, 1994, pp. 902-906.

[3] B. Kim and S. Park, Characterization of inductively coupled plasma using neural networks, IEEE Transactions on Plasma Science, Vol. 30, 2002, pp. 698-705.

[4] G. Erten, A. Gharbi, F. Salam, T. Grotjohn and J. Asmussen, "Using neural networks to control the process of plasma etching and deposition", IEEE Proceedings of International Conference on Neural Networks, 1996, pp. 1091-1096.

[5] D. Stokes and G.S. May, Real-time control of reactive ion etching using neural networks, IEEE Transactions on Semiconductor Manufacturing. Vol. 13, 2000, pp. 469-480.

[6] J. VanAntwerp, R. Braatz and R. Sahinidis, Robust nonlinear control of plasma etching, Electrochemical proceedings, Vol. 10, 1997, pp. 454462.

[7] G. Balas, J. Doyle, K. Glover, A. Packard, R. Smith, $\mu$-Analysis and Synthesis Toolbox, User's Guide, The MathWorks Inc., Natick. 1993.

[8] S. Skogestad, and I. Postlethwaite, Multivariable Feedback Control : Analysis and Design, Wiley, London, 1996.

[9] M. Turner, DCU simple plasma model. private communication, 2003.

[10] P. Iordanov, Modelling and analysis of a simple plasma process, technical report EE/JVR/4/04, NUI Maynooth, Ireland, 2004.

[11] P. Iordanov, J. Ringwood and S. Doherty, "Control problem classification for a plasma process", Proceedings of the 16th IFAC World Congress, Prague, Czech Republic, 2005.

[12] J. Doyle, Analysis of feedback systems with structured uncertainties. In: IEE Proceedings. Vol. 129, 1982, pp. 242-250. 